\title{
Regional Anti-windup Compensation for Linear Systems with Input Saturation
}

\author{
Tingshu Hu, Andrew R. Teel and Luca Zaccarian
}

\begin{abstract}
In this paper we propose LMI methods for designing dynamic/static anti-windup compensators to improve regional performance and stability of linear control systems with saturating actuators. Algorithms are developed for minimizing the upper bound on the regional $\mathcal{L}_{2}$ gain for exogenous inputs with $\mathcal{L}_{2}$ norm bounded by a given value, and for minimizing this upper bound with a guaranteed reachable set or domain of attraction. Based on the structure of the optimization problems, it is shown that for systems whose plants have poles in the closed left-half plane, plant order dynamic anti-windup can achieve semiglobal stability and finite $\mathcal{L}_{2}$ gain for exogenous inputs with $\mathcal{L}_{2}$ norm bounded by any finite value. The problems are studied in a general setting where the only requirement on the linear control system is well-posedness and internal stability.
\end{abstract}

keywords: anti-windup synthesis, $\mathcal{L}_{2}$ gain, reachable set, domain of attraction, LMIs

\section{INTRODUCTION}

Anti-windup compensation has evolved from mere heuristics at its early stage of development, to systematic design procedures based on various optimization tools in the last decade. More recently, some structural interpretation about the achievable performance of linear anti-windup for linear saturated control systems has been revealed through rigorous theoretical analysis. Over the last decade, systematic approaches have been proposed based on robustness and $H_{\infty}$ optimal control [4], [5], [6], [8], [15], [18], and extensive numerical design algorithms have been developed based on LMI optimization tools [1], [2], [7], [3], [9], [11], [10], [16], [17], [20]. Among these papers, [17] studied the general case where the controller is dynamic, the exogenous input directly enters the actuator and there is a correction term in the output equation of the controller. In [17], static anti-windup compensators were constructed for global stabilization and reduced $\mathcal{L}_{2}$ gain performance. These synthesis problems were cast for the first time as convex optimization problems with LMI constraints for the general configuration.

The recent work [9] reached further by proposing an LMI-based synthesis method for the construction of dynamic anti-windup compensators guaranteeing finite $\mathcal{L}_{2}$ gain on control systems with exponentially stable plants.

Work supported in part by AFOSR grant number F49620- 03-1-0203, NSF under Grants ECS-9988813 and ECS-0324679, by ENEA-Euratom, ASI and MIUR under PRIN and FIRB projects.

A.R. Teel is with the Department of Electrical and Computer Engineering, University of California, Santa Barbara, CA 93106, USA teel aece.ucsb. edu

T. Hu is with the Dept. of ECE, Umass Lowell, Lowell, MA 01854, USA tingshu_hu@uml . edu

L. Zaccarian is with the Dipartimento di Informatica, Sistemi e Produzione, University of Rome, Tor Vergata, 00133 Rome, Italy zack@disp. uniroma2 . it
It was concluded that, for this class of systems, the global $\mathcal{L}_{2}$ gain can always be made finite by designing the dynamic anti-windup compensator with the algorithm developed in the paper. This conclusion promises global stability before the anti-windup compensator is constructed, even before the linear controller is designed, thus providing full confidence in designing a linear controller for the best local performances.

While the boundedness of the global $\mathcal{L}_{2}$ gain provides a guaranteed global performance of the closed-loop system, it might be conservative for practical situations where the $\mathcal{L}_{2}$ norm of the exogenous input is bounded below a known constant. Moreover, for non-exponentially-stable plants, global finite $\mathcal{L}_{2}$ gain cannot be achieved through bounded inputs and it becomes of high interest to determine a regional finite $\mathcal{L}_{2}$ gain for a class of norm bounded inputs. These situations motivate us to characterize the nonlinear $\mathcal{L}_{2}$ gain for general systems with anti-windup augmentation and to design an anti-windup compensator to minimize the $\mathcal{L}_{2}$ gain for a class of norm bounded inputs.

An attempt to characterize the $\mathcal{L}_{2}$ gain for norm bounded inputs has been made in [19]. The main result is based on the assumption that the deadzone function lies within a sector $[0, K]$ with $K<I$ during the operation of the system. However, for the general case where the exogenous input directly enters the actuator, exogenous inputs with arbitrarily small $\mathcal{L}_{2}$ norm may yield actuator inputs with arbitrarily large $\mathcal{L}_{\infty}$ norm. Hence there exists no $K<$ $I$ such that the deadzone function can be bounded by $[0, K]$. It is therefore clear that, for a general anti-windup configuration, the idea of using a narrowed sector to replace the global sector $[0, I]$ for regional performance analysis will not go through.

In our concurrent paper [14], we developed regional analysis tools for characterization of the nonlinear $\mathcal{L}_{2}$ gain and the reachable set for a class of norm bounded inputs, and for the estimation of the domain of attraction for general anti-windup systems where the only assumptions are well-posedness and local stability. The regional analysis results were based on a flexible description of the saturation/deadzone function in terms of an auxiliary linear function of the state. This description generalizes the tools developed in [13], [12] for stability analysis. Because of this flexible description, all the regional analysis results in [14] were able to be presented in the form of LMIs.

In this paper, we will propose design methods for the construction of dynamic anti-windup compensators that optimize the regional $\mathcal{L}_{2}$ gain of the resulting closed-loop. Our results correspond to the generalization of the constructions in [9], where global $\mathcal{L}_{2}$ gain was enforced on the closed- 
loop system (so that the approach was only applicable when dealing with exponentially stable linear plants). Moreover, our results generalizes the LMI approach for static antiwindup design proposed in [7] also to the dynamic case, so that the construction of [7] leads to our same results when setting the anti-windup state size to zero. ${ }^{1}$ Similar to [9], we provide LMI-based constructions for static and plant order anti-windup designs. These designs will lead to regional results if the plant is exponentially unstable and to semi-global results if the plant is marginally unstable (in both of these cases, global results are not achievable with linear compensation).

This paper is organized as follows: In Section II we formulate the problems that will be addressed in the paper; in Section III we state the LMI-based feasibility conditions for regional anti-windup design; in Section IV we give the procedure for the anti-windup construction (which is based on the solution to the feasibility conditions); in Section V we illustrate the proposed constructions on a numerical example.

Notation For compact presentation of matrices, given a square matrices $X$ we denote $\operatorname{He} X:=X+X^{T}$. For $P=P^{T}>0$, denote $\mathcal{E}(P):=\left\{x: x^{T} P x \leq 1\right\}$. We will call a linear system "marginally unstable" if it has poles on the imaginary axis but has no poles in the open right half plane.

\section{Problem STATEMENT}

Consider a linear plant,

$$
\mathcal{P}\left\{\begin{array}{l}
\dot{x}_{p}=A_{p} x_{p}+B_{p, u} u+B_{p, w} w \\
y=C_{p, y} x_{p}+D_{y, y u} u+D_{p, y w} w \\
z=C_{p, z} x_{p}+D_{p, z u} u+D_{p, z w} w
\end{array}\right.
$$

where $x_{p} \in \mathbb{R}^{n_{p}}$ is the plant state, $u \in \mathbb{R}^{n_{u}}$ is the control input, $w \in \mathbb{R}^{n_{w}}$ is the exogenous input (possibly containing disturbance, reference and measurement noise), $y \in \mathbb{R}^{n_{y}}$ is the measurement output and $z \in \mathbb{R}^{n_{z}}$ is the performance output.

Assume that an unconstrained controller has been designed,

$$
\mathcal{C}\left\{\begin{array}{l}
\dot{x}_{c}=A_{c} x_{c}+B_{c, u} y+B_{c, w} w+v_{1}, \\
y_{c}=C_{c} x_{c}+D_{c, u} y+D_{c, w} w+v_{2},
\end{array}\right.
$$

where $x_{c} \in \mathbb{R}^{n_{c}}$ is the controller state and $y_{c} \in \mathbb{R}^{n_{u}}$ is the controller output, $v_{1}$ and $v_{2}$ will be used for anti-windup augmentation. In the case without plant input saturation (therefore, without any anti-windup compensation), the socalled unconstrained closed-loop is formed by setting

$$
u=y_{c}, \quad v_{1}=0, v_{2}=0 .
$$

In our study we assume that the unconstrained closed-loop system has the following property.

Assumption 1: The unconstrained closed-loop system (1), (2), (3) is well posed and internally stable.

\footnotetext{
${ }^{1}$ Note however that the approach in [7] does not lead to LMIs when used for the dynamic case, whereas our approach leads to convex tools both for the static and for the plant order anti-windup compensation design. In addition, the case where the plant has a feedthrough term is not covered in [7], whereas we address that case, and the arising algebraic loop implications, in this paper.
}

In the presence of actuator saturation, the relation between $u$ and $y_{c}$ is described as $u=\operatorname{sat}\left(y_{c}\right)$, where $\operatorname{sat}(\cdot)$ : $\mathbb{R}^{n_{u}} \rightarrow \mathbb{R}^{n_{u}}$ is a saturation function with its $i$ th component depending on the $i$ th input component $y_{c i}$ as follows:

$$
\operatorname{sat}_{\bar{u}_{i}}\left(y_{c i}\right)= \begin{cases}\bar{u}_{i}, & \text { if } y_{c i} \geq \bar{u}_{i} \\ y_{c i}, & \text { if }-\bar{u}_{i} \leq y_{c i} \leq \bar{u}_{i} \\ -\bar{u}_{i}, & \text { if } y_{c i} \leq-\bar{u}_{i} .\end{cases}
$$

To avoid or to minimize performance degradation caused by saturation, the closed-loop system will be augmented with the following anti-windup compensator

$$
\mathcal{A} \mathcal{W}\left\{\begin{aligned}
\dot{x}_{a w} & =A_{a w} x_{a w}+B_{a w}\left(y_{c}-\operatorname{sat}\left(y_{c}\right)\right) \\
v & =C_{a w} x_{a w}+D_{a w}\left(y_{c}-\operatorname{sat}\left(y_{c}\right)\right),
\end{aligned}\right.
$$

where $x_{a w} \in \mathbb{R}^{n_{a w}}, v=\left[\begin{array}{l}v_{1} \\ v_{2}\end{array}\right] \in \mathbb{R}^{n_{c}+n_{u}}$, and the unconstrained interconnection (3) is replaced by the following anti-windup interconnection

$$
u=\operatorname{sat}\left(y_{c}\right)
$$

The resulting nonlinear closed-loop (1), (2), (5), (6) is depicted in Figure 1 and will be denoted anti-windup closed-loop henceforth.

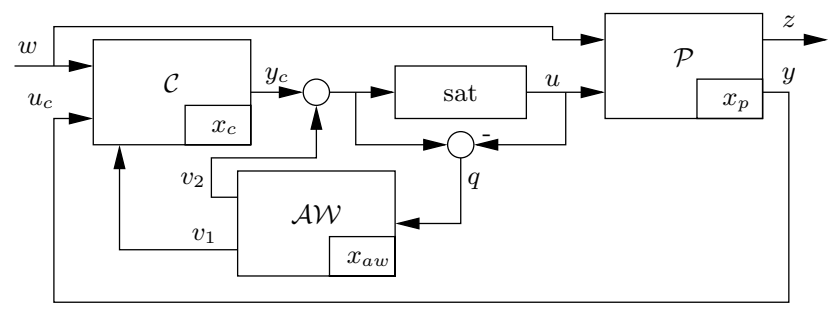

Fig. 1. The linear anti-windup closed-loop system.

Denote $x_{c l}=\left[\begin{array}{cc}x_{p}^{T} & x_{c}^{T}\end{array}\right]^{T}$. The configuration in Fig. 1 can be described by the following equation

$$
\begin{aligned}
& \dot{x}_{c l}=A_{c l} x_{c l}+B_{c l, w} w+B_{c l, q} q+B_{c l, v} v \\
& z=C_{c l, z} x_{c l}+B_{c l, z w} w+B_{c l, z q} q+B_{c l, z v} v \\
& y_{c}=C_{c l, y} x_{c l}+B_{c l, y w} w+B_{c l, y q} q+B_{c l, y v} v
\end{aligned}
$$

where $v$ depends on $q:=y_{c}-\operatorname{sat}\left(y_{c}\right)$ through the dynamics of the anti-windup. Here all the matrices are uniquely determined by those of the plant (1) and the controller (2).

For the system in Fig. 1, an LMI-based synthesis method was developed in [9] for designing dynamic anti-windup compensators to minimize an upper bound of the global $\mathcal{L}_{2}$ gain (the approach being therefore only applicable to the case where the plant is exponentially stable). The objective of this paper and our concurrent work [14] is to develop synthesis tools for general control systems whose plant may be not exponentially stable. For these systems, linear antiwindup cannot induce global finite $\mathcal{L}_{2}$ gain. Therefore, we need to restrict our attention to a class of inputs $w$ whose $\mathcal{L}_{2}$ norm is bounded by a given value, i.e., $\|w\|_{2} \leq s$ for a given $s$. Due to this restriction, not only it becomes of interest to minimize the arising regional $\mathcal{L}_{2}$ gain, but also to provide guarantees both on the reachable set from such 
a bounded input and on the domain of attraction ensured by the corresponding anti-windup compensator. We will therefore address design problems reported next, where we denote the closed-loop state by $x:=\left[x_{p}^{T} x_{c}^{T} x_{a w}^{T}\right]^{T}$ and its dimension by $n:=n_{p}+n_{c}+n_{a w}$.

Problem 1: Consider the plant (1), the controller (2) and a bound $s$ on $\|w\|_{2}$. Design an anti-windup compensator (5) such that the relation

$$
\|z\|_{2} \leq \gamma\|w\|_{2}, \quad \forall w \text { such that }\|w\|_{2} \leq s, x(0)=0
$$

is satisfied with a minimal $\gamma$.

Problem 2: Consider the plant (1), the controller (2) and a bound $s$ on $\|w\|_{2}$. Given a desired reachable set $\mathcal{R}_{p} \subset$ $\mathbb{R}^{n_{p}}$. Design an anti-windup compensator (5) such that with $x(0)=0$ and $\|w\|_{2} \leq s$, we have $x_{p}(t) \in \mathcal{R}_{p}$ for all $t>0$ while (10) is satisfied with a minimal $\gamma$.

Problem 3: Consider the plant (1), the controller (2) and a bound $s$ on $\|w\|_{2}$. Given a desired stability region $\mathcal{S}_{p} \subset$ $\mathbb{R}^{n_{p}}$. Design an anti-windup compensator such that for all $x_{p}(0) \in \mathcal{S}_{p}$, there exist $x_{c}(0) \in \mathbb{R}^{n_{c}}, x_{a w}(0) \in \mathbb{R}^{n_{a w}}$ to make $\lim _{t \rightarrow \infty} x(t)=0$ while (10) is satisfied with a minimal $\gamma$.

\section{REGIONAL ANTI-WINDUP DESIGN: FEASIBILITY}

In this section present a set of feasibility conditions for solving Problems 1 to 3 . The construction of anti-windup compensators which solve each of the problems will be provided in Section IV. We first address the feasibility problem of anti-windup design for optimal $\mathcal{L}_{2}$ gain from bounded inputs, then augment the problem with extra requirements such as guaranteed reachable set and guaranteed domain of attraction. As with the global results of [9], the regional results would involve nonconvex conditions for a generic order of the anti-windup compensator but reduce to convex conditions when the anti-windup compensator is static or of the same order as that of the plant.

\section{A. Optimal $\mathcal{L}_{2}$ gain for bounded inputs}

The following theorem establishes feasibility conditions corresponding to Problem 1 for the plant order case and the static case, respectively.

Theorem 1: Consider the plant (1) and the controller (2) satisfying Assumption 1. Assume that $x_{p}(0)=0, x_{c}(0)=0$ and $\|w\|_{2} \leq s$.

1) an optimal plant-order anti-windup compensator solving Problem 1 can be constructed based on the optimal solution $\left(R_{11}, S, Z, \gamma^{2}\right)$ to the following LMIoptimization problem in the variables $R_{11}=R_{11}^{T}>$ $0, S=S^{T}:=\left[\begin{array}{ll}S_{11} & S_{12} \\ S_{12}^{T} & S_{22}\end{array}\right]>0, \gamma^{2}>0, Z$, whenever the corresponding constraints are feasible:

$$
\begin{aligned}
& \min _{Z, R_{11}, S, \gamma^{2}} \gamma^{2}, \text { subject to } \\
& \operatorname{He}\left[\begin{array}{ccc}
A_{p} R_{11}+B_{p, u} Z & B_{p, w} & 0 \\
0 & -\frac{I}{2} & 0 \\
C_{p, z} R_{11}+D_{p, z u} Z & D_{p, z w} & -\frac{\gamma^{2} I}{2}
\end{array}\right]<0
\end{aligned}
$$

$$
\begin{aligned}
& \operatorname{He}\left[\begin{array}{ccc}
A_{c l} S & B_{c l, w} & 0 \\
0 & -\frac{I}{2} & 0 \\
C_{c l, z} S & D_{c l, z w} & -\frac{\gamma^{2} I}{2}
\end{array}\right]<0 \\
& R_{11}-S_{11}>0 . \\
& {\left[\begin{array}{cc}
\bar{u}_{i}^{2} / s^{2} & Z_{i} \\
Z_{i}^{T} & R_{11}
\end{array}\right] \geq 0, \quad i=1, \ldots, n_{u},}
\end{aligned}
$$

where $Z_{i}$ denotes the $i$ th row of $Z$;

2) an optimal static anti-windup compensator solving Problem 1 can be constructed based on the optimal solution $\left(R, Z, \gamma^{2}\right)$ to the following LMI-optimization problem in the variables $R=R^{T}:=\left[\begin{array}{ll}R_{11} & R_{12} \\ R_{12}^{T} & R_{22}\end{array}\right]>0$, $\gamma^{2}>0, Z$, whenever the corresponding constraints are feasible:

$$
\begin{aligned}
& \min _{Z, R, \gamma^{2}} \gamma^{2} \text {, subject to } \\
& (11 a),(11 d) \\
& \operatorname{He}\left[\begin{array}{ccc}
A_{c l} R & B_{c l, w} & 0 \\
0 & -\frac{I}{2} & 0 \\
C_{c l, z} R & D_{c l, z w} & -\frac{\gamma^{2}}{2}
\end{array}\right]<0
\end{aligned}
$$

Remark 1: (Lower bounds on performance level) Paralleling [9, Remark 7], conditions (11a) and (11b) can be interpreted based on the LMI formulation of the bounded real lemma (which is also reported in [9, Lemma 1]). Indeed, as in [9], condition (11b) constrains $\gamma$ to be not smaller than the $\mathcal{L}_{2}$ gain from $w$ to $z$ of the unconstrained closed-loop. Moreover, by selecting $Z=K_{p} R_{11}$, (11a) corresponds to the $\mathcal{L}_{2}$ gain from $w$ to $z$ for the plant controlled by a state feedback law $u=K_{p} x_{p}$. Therefore, (11a) constrains $\gamma$ to be not smaller than the $\mathcal{L}_{2}$ gain of the compensated plant by way of this extra stabilizing action. Note however that $K_{p}$ in the corresponding feedback law is further constrained by (11d), so that the open-loop plant $\mathcal{L}_{2}$ gain may be only reduced to a certain extent when larger values of $s$ make the constraint (11d) tighter. As $s$ approaches $+\infty, Z$ will approach 0 and the constraint on $\gamma$ enforced by (11a) will approach the global constraint commented in [9] and corresponding to the $\mathcal{L}_{2}$ gain of the open-loop plant.

For a system with an exponentially unstable plant (to rule out defective cases, assume that the exponentially unstable modes are observable from the output $z$ and controllable from the exogenous input $w$ ), it is clear that there exists a norm bounded $w$ such that $\|z\|_{2}$ is unbounded and thus (11a-d) is feasible only for $s$ bounded by a certain $\bar{s}$. On the other hand, if $A_{p}$ is Hurwitz, then there exists a selection $\left(R_{11}, S, Z, \gamma^{2}\right)$ with $Z=0$ satisfying the LMIs in (11) for all $s$ (this solution corresponds to the global construction proposed in [9]). The critical case where $A_{p}$ has eigenvalues on the imaginary axis and in the left half plane was not addressed in [9] and can be dealt with here with arbitrarily large selections of $s$.

Proposition 1: Consider the plant (1) and the controller (2) satisfying Assumption 1. Suppose that the plant is not exponentially unstable. Then for each $s>0$, the constraints (11a-d) are feasible with a finite $\gamma$.

However, unlike the exponentially stable case, there doesn't exist a single selection satisfying the LMIs in 
(11) for all $s$ (this would be impossible, as finite global $\mathcal{L}_{2}$ gain cannot be achieved with bounded inputs on a marginally unstable plant). As $s$ increases, new solutions $\left(R_{11}, S, Z, \gamma^{2}\right)$ will be feasible with increased $\gamma$.

\section{B. Optimal $A W$ with guaranteed reachable set}

In this section we augment the design problem of the previous section with the extra requirement that given a bound $s$ on the $\mathcal{L}_{2}$ norm of $w$, the plant state does not exit a given desirable set $\mathcal{R}_{p} \subset \mathbb{R}^{n_{p}}$. We first pick

$$
\mathcal{R}_{p}=\mathcal{E}\left(R_{p}^{-1}\right)=\left\{x_{p}: x_{p}^{T} R_{p}^{-1} x_{p} \leq 1\right\},
$$

where $R_{p}=R_{p}^{T}>0$ and will then explain how the result can be extended to other types of sets $\mathcal{R}_{p}$.

Theorem 2: Consider the plant (1) and the controller (2) satisfying Assumption 1. Assume that $x_{p}(0)=0, x_{c}(0)=0$ and $\|w\|_{2} \leq s$. Then

1) an optimal plant-order anti-windup compensator solving Problem 2 can be constructed based on the optimal solution $\left(R_{11}, S, Z, \gamma^{2}\right)$ to the following LMIoptimization problem in the variables $R_{11}=R_{11}^{T}>0$, $S=S^{T}:=\left[\begin{array}{ll}S_{11} & S_{12} \\ S_{12}^{T} & S_{22}\end{array}\right]>0, \gamma^{2}>0, Z$, whenever the corresponding constraints are feasible:

$$
\begin{aligned}
& \min _{Z, S, R_{11}, \gamma^{2}} \gamma^{2}, \text { subject to } \\
& (11 a),(11 b),(11 c),(11 d), \\
& s^{2} R_{11} \leq R_{p}
\end{aligned}
$$

2) an optimal static anti-windup compensator solving Problem 2 can be constructed based on the optimal solution $\left(R, Z, \gamma^{2}\right)$ to the following LMI-optimization problem in the variables $R=R^{T}:=\left[\begin{array}{ll}R_{11} & R_{12} \\ R_{12}^{T} & R_{22}\end{array}\right]>0$, $\gamma^{2}>0, Z$, whenever the corresponding constraints are feasible:

$$
\begin{aligned}
& \min _{Z, R, \gamma^{2}} \gamma^{2}, \text { subject to } \\
& (11 a),(12 b),(11 d), \\
& s^{2} R_{11} \leq R_{p} .
\end{aligned}
$$

The main idea behind the results of Theorem 2 relies on the fact that if (14a) (respectively, (15a)) is satisfied, then there exists an anti-windup compensator such that the reachable set is bounded by $\mathcal{E}\left(\left(s^{2} R_{11}\right)^{-1}\right)$. Moreover, the constraint (14b) (respectively, (15b)) implies that this set is inside the desired reachability set given by (13), namely that $\mathcal{E}\left(\left(s^{2} R_{11}\right)^{-1}\right) \subset \mathcal{E}\left(R_{p}^{-1}\right)$.

Based on Theorem 2, we can also formulate optimization problems to minimize the desirable reachable set $\mathcal{R}_{p}$ under the constraints (14a) and (14b), with a guaranteed $\mathcal{L}_{2}$ gain $\gamma$ (or without considering the $\mathcal{L}_{2}$ gain). The quantity to be minimized can be the trace of $R_{p}$ or the determinant of $R_{p}$. We may also take $\mathcal{R}_{p}$ as the following unbounded set:

$$
\mathcal{R}_{p}(\alpha)=\left\{x_{p}:|C x| \leq \alpha\right\}
$$

where $C \in \mathbb{R}^{1 \times n_{p}}$ is a given row vector. Then $\mathcal{E}\left(\left(s^{2} R_{11}\right)^{-1}\right) \subset \mathcal{R}_{p}(\alpha)$ if and only if $C R_{11} C^{T} \leq \alpha^{2} / s^{2}$. If both (14a) and $C R_{11} C^{T} \leq \alpha^{2} / s^{2}$ are enforced to hold in the LMI optimization, then it follows that $\left|C x_{p}(t)\right| \leq \alpha$ for all $t$ if $\|w\|_{2} \leq s$. Therefore, if our objective is to minimize the maximum value of a particular output $C x_{p}$, we may formulate the following optimization problem:

$$
\begin{aligned}
& \min _{Z, S, R_{11}, \alpha} \alpha, \text { subject to } \\
& (11 a),(11 b),(11 c),(11 d), \\
& C R_{11} C^{T}<\alpha^{2} / s^{2},
\end{aligned}
$$

where a desirable $\mathcal{L}_{2}$ gain can be incorporated in ( 16a). If there is no consideration for a desirable $\mathcal{L}_{2}$ gain, then the matrices in (16) can be simplified by removing the third block row and the third block column of the matrices in (11a) and (11b). (A similar approach could also be derived when using static anti-windup design.)

\section{Optimal AW with guaranteed domain of attraction}

We now augment the design problem of Section III-A for the purpose of solving Problem 3 with a guaranteed domain of attraction in terms of the state of the plant:

$$
\mathcal{S}_{p}=\mathcal{E}\left(S_{p}^{-1}\right)=\left\{x_{p}: x_{p}^{T} S_{p}^{-1} x_{p} \leq 1\right\},
$$

where $S_{p}=S_{p}^{T}>0$.

Theorem 3: Consider the plant (1) and the controller (2) satisfying Assumption 1.

1) an optimal plant-order anti-windup compensator solving Problem 3 can be constructed based on the optimal solution $\left(R_{11}, S, Z, \gamma^{2}\right)$ to the following LMIoptimization problem in the variables $R_{11}=R_{11}^{T}>0$, $S=S^{T}:=\left[\begin{array}{ll}S_{11} & S_{12} \\ S_{12}^{T} & S_{22}\end{array}\right]>0, \gamma^{2}>0, Z$, whenever the corresponding constraints are feasible:

$$
\begin{aligned}
& \min _{Z, S, R_{11}, \gamma^{2}} \gamma^{2}, \text { subject to } \\
& (11 a),(11 b),(11 c),(11 d), \\
& s^{2} R_{11} \geq S_{p}
\end{aligned}
$$

2) an optimal static anti-windup compensator solving Problem 3 can be constructed based on the optimal solution $\left(R, Z, \gamma^{2}\right)$ to the following LMI-optimization problem in the variables $R=R^{T}:=\left[\begin{array}{ll}R_{11} & R_{12} \\ R_{12}^{T} & R_{22}\end{array}\right]>0$, $\gamma^{2}>0, Z$, whenever the corresponding constraints are feasible:

$$
\begin{aligned}
& \min _{Z, R, \gamma^{2}} \gamma^{2}, \text { subject to } \\
& (11 a),(12 b),(11 d), \\
& s^{2} R_{11} \geq S_{p}
\end{aligned}
$$

Based on Theorem 3, we can formulate various optimization problems to maximize the estimate of the domain of attraction (with respect to different measures of set size) with a guaranteed $\mathcal{L}_{2}$ gain (or without considering the $\mathcal{L}_{2}$ gain).

If the plant is exponentially stable, then global asymptotic stability by dynamic anti-windup compensation is guaranteed by the finite global $\mathcal{L}_{2}$ gain [9]. If the plant is exponentially unstable, then only local stability can be guaranteed. For a plant that is marginally unstable, what may be desired is semiglobal stability with respect to the 
state of the plant. This is guaranteed when using plant order anti-windup by the following proposition.

Proposition 2: Consider the plant (1) and the controller (2) satisfying Assumption 1. Suppose that the plant is not exponentially unstable. Then, for any finite $S_{p}$ and for any $s>0,(18 \mathrm{a})$ and (18b) are feasible.

\section{Regional ANTI-WINDUP DESIGN: CONSTRUCTION}

In this section, based on the solutions to the feasibility conditions listed in Section III, we provide a constructive algorithm for determining the matrices of a plant order dynamic anti-windup compensator. The construction of a static compensator is much simpler and will be suggested in square brackets.

The algorithm follows from the proof of Theorems 1 to 3. It is based on the solution $\left(R_{11}, S, Z, \gamma^{2}\right)\left[\left(R, Z, \gamma^{2}\right)\right]$ to the plant-order [static] anti-windup feasibility conditions. Note that the anti-windup matrices constructions are the same regardless of what optimization problem the feasibility matrices arise from (this may be the one listed in Theorems 1, 2 or 3 or any modification of them). Note also that the algorithm is similar to the ones reported in [9] (except for the use of the variable $Z$ ).

Procedure 1: (Anti-windup construction)

Step 1. Solve the feasibility LMIs. Find a solution $\left(R_{11}, S, Z, \gamma^{2}\right)\left[\left(R, Z, \gamma^{2}\right)\right]$ to the feasibility LMIs listed in Section III.

Step 2. Construct the matrix $Q$. Define the matrices $R:=\left[\begin{array}{ll}R_{11} & S_{12} \\ S_{12}^{T} & S_{22}\end{array}\right]$ and $N \in \mathbb{R}^{\left(n_{p}+n_{c}\right) \times n_{a w}}$ as a solution of the following equation:

$$
R S^{-1} R-R=N N^{T} .
$$

Since $R$ and $S$ are invertible and by the feasibility conditions, $R S^{-1} R-R$ is positive semidefinite and of rank $n_{a w}$. Hence there always exists a matrix $N$ satisfying equation (20). Define the matrix $M \in \mathbb{R}^{n_{a w} \times n_{a w}}$ as

$$
M:=I+N^{T} R^{-1} N .
$$

Finally, define the matrix $Q \in \mathbb{R}^{n \times n}\left(n=n_{p}+n_{c}+\right.$ $\left.n_{a w}\right)$ as

$$
Q:=\left[\begin{array}{cc}
R & N \\
N^{T} & M
\end{array}\right] .
$$

[Define the matrix $Q=R$.]

Step 3. Build necessary matrices. Construct the matrices $A_{0} \in \mathbb{R}^{n \times n}, B_{0} \in \mathbb{R}^{n \times n_{u}}, C_{y 0} \in \mathbb{R}^{n_{u} \times n}$, $D_{y q 0} \in \mathbb{R}^{n_{u} \times n_{u}}, C_{z 0} \in \mathbb{R}^{n_{z} \times n}, D_{z q 0} \in \mathbb{R}^{n_{z} \times n_{u}}$, $B_{w} \in \mathbb{R}^{n \times n_{w}}, D_{z w} \in \mathbb{R}^{n_{z} \times n_{w}}$ and $D_{y w} \in \mathbb{R}^{n_{u} \times n_{w}}$ as

$$
\left[\begin{array}{c|c|c}
A_{0} & B_{q 0} & B_{w} \\
\hline C_{y 0} & D_{y q 0} & D_{y w} \\
\hline C_{z 0} & D_{z q 0} & D_{z w}
\end{array}\right]=\left[\begin{array}{cc|c|c}
A_{c l} & 0 & B_{c l, q} & B_{c l, w} \\
0 & 0 & 0 & 0 \\
\hline C_{c l, y} & 0 & D_{c l, y q} & D_{c l, y w} \\
\hline C_{c l, z} & 0 & D_{c l, z q} & D_{c l, z w}
\end{array}\right]
$$

[Define the matrices above by removing the second block row and block column of zeros from the right hand side of the above equation.]
Step 4. Anti-windup compensator LMI. Based on Steps 2 and 3, construct the matrices $H \in \mathbb{R}^{\left(n_{a w}+n_{c}+n_{u}\right) \times\left(n+n_{u}+n_{w}+n_{z}\right)}, \quad \Psi_{R} \in$ $\mathbb{R}^{\left(n+n_{u}+n_{w}+n_{z}\right) \times\left(n+n_{u}+n_{w}+n_{z}\right)}$ and $G_{U} \in \mathbb{R}^{\left(n_{a w}+n_{u}\right) \times\left(n+n_{u}+n_{w}+n_{z}\right)}$ as follows:

$$
\begin{gathered}
\Psi_{R}=\operatorname{He}\left[\begin{array}{cccc}
A_{0} Q & B_{q 0} U-Y^{T} & B_{w} & Q C_{z 0}^{T} \\
C_{y 0} Q & D_{y q 0} U-U & D_{y w} & U D_{z q 0}^{T} \\
0 & 0 & -\frac{I}{2} I & D_{z w}^{T} \\
0 & 0 & 0 & -\frac{\gamma^{2}}{2} I
\end{array}\right], \\
H=\left[\begin{array}{cc|c|c|c}
0 & I_{n_{a w}} & 0 & 0 & 0 \\
B_{c l, v}^{T} & 0 & D_{c l, y v}^{T} & 0 & D_{c l, z v}^{T}
\end{array}\right] \\
G_{U}=\left[\begin{array}{cc|c|c|c}
N^{T} & M & 0 & 0 & 0 \\
0 & 0 & I & 0 & 0
\end{array}\right],
\end{gathered}
$$

where $Y \in \mathbb{R}^{n_{u} \times n_{p}+n_{c}+n_{a w}}$ is defined as $Y:=$ [ $\left.Z Z R_{11}^{-1} R_{12} Z R_{11}^{-1} N_{1}\right]$ (where $N_{1}$ is the upper block of the matrix $N)\left[Y:=\left[\begin{array}{ll}Z & Z R_{11}^{-1} R_{12}\end{array}\right]\right.$. Finally, solve the LMI

$$
\Psi_{R}+G_{U}^{T} \Lambda_{U}^{T} H+H^{T} \Lambda_{U} G_{U}<0 .
$$

in the unknowns $\Lambda_{U} \in \mathbb{R}^{\left(n_{a w}+n_{c}+n_{u}\right) \times\left(n_{a w}+n_{u}\right)}$ and $U \in \mathbb{R}^{n_{u} \times n_{u}}, U>0$ diagonal, and compute the matrices of the anti-windup compensator (5) as follows:

$$
\left[\begin{array}{cc}
A_{a w} & B_{a w} \\
C_{a w} & D_{a w}
\end{array}\right]=\Lambda_{U}\left[\begin{array}{cc}
I & 0 \\
0 & U^{-1}
\end{array}\right]
$$

[compute the static anti-windup gain as $D_{a w}=$ $\left.\Lambda_{U} U^{-1}\right]$.

\section{EXAMPLES}

Example 1: We adopt Example 2 from [9]. The plant is a cart-spring-pendulum system with one control input, one disturbance input, four states and one measurement output. The plant state is $x_{p}=\left[\begin{array}{llll}p & \dot{p} & \theta & \dot{\theta}\end{array}\right]$, where $p$ is the horizontal displacement of the cart and $\theta$ is the angle of the pendulum. The plant and controller parameters can be found in [9]. For this example, the closed-loop system without anti-windup compensation is not globally stable. Also, there exists no static anti-windup compensation to make the global $\mathcal{L}_{2}$ gain bounded. With dynamic antiwindup augmentation, an upper bound for the achievable global $\mathcal{L}_{2}$ gain is found to be 181.1424 .

The achievable $\mathcal{L}_{2}$ gain for every $s>0$ by using plant order anti-windup can be determined with the algorithm based on Theorem 1 . By choosing different $s$ over $(0, \infty)$, the achievable performance can be obtained as a function of $s$. Fig. 2 plot this achievable performance in solid curve. For comparison, we also plot the the achievable performance by using static anti-windup, which is the dashed-dotted curve in Fig. 2. Also plotted in Fig. 2 (dashed) is the upper bound for the nonlinear $\mathcal{L}_{2}$ gain under a particular plant order antiwindup compensator.

Next, we use algorithm (16) to determine an achievable upper bound on the displacement of the cart $x_{p 1}$ (by plant order anti-windup) for a given norm bound $s$ on $\|w\|_{2}$. For this purpose, we choose $C=\left[\begin{array}{llll}1 & 0 & 0 & 0\end{array}\right]$. The 


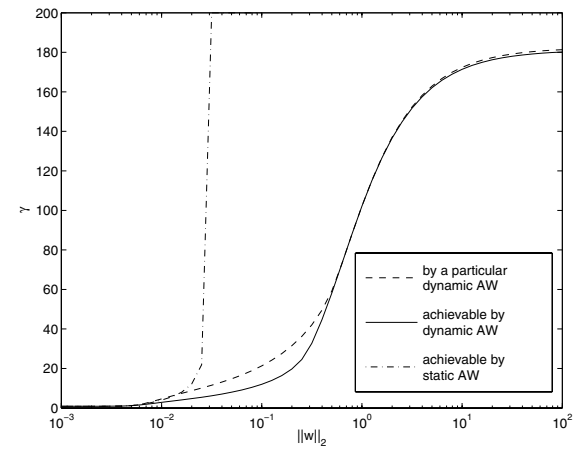

Fig. 2. Achievable nonlinear $\mathcal{L}_{2}$ gains for Example 1.

relation between $s$ and the achievable bound $\alpha$ is plotted in Figure 3. If we take $C=\left[\begin{array}{llll}0 & 0 & 1 & 0\end{array}\right]$, then we obtain an achievable upper bound on the angle of the pendulum $x_{p 3}$. The relation between $s$ and the achievable bound $\alpha$ for $x_{p 3}$ is plotted in Figure 4.

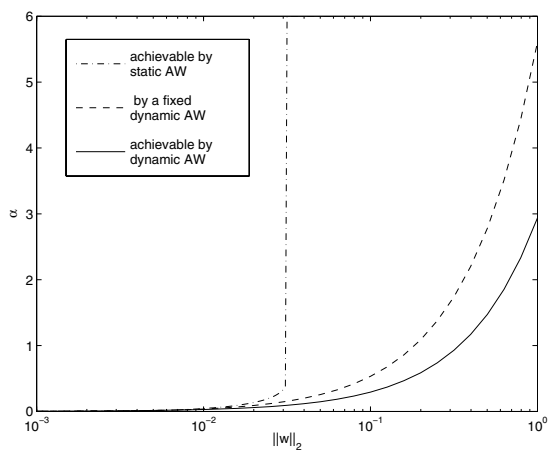

Fig. 3. Achievable bounds on $x_{p 1}$.

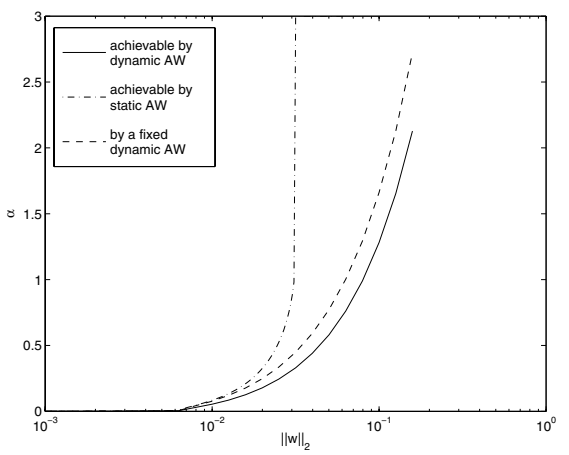

Fig. 4. Achievable bounds on $x_{p 3}$.

\section{CONCLUSIONS}

In this paper we proposed synthesis tools for the construction of linear anti-windup compensators for a variety of regional stability and performance goals. The problems are formulated in a general setup with linear dynamic controllers and linear dynamic anti-windup compensators, where the only assumption on the closed-loop linear system is well-posedness and internal stability. Solutions to the problems are presented through a set of convex optimization procedures based on LMI constraints. Furthermore, two semiglobal results have been established for the special case where the plant is marginally unstable.

\section{REFERENCES}

[1] Y.Y. Cao, Z. Lin, and D.G. Ward. An antiwindup approach to enlarging domain of attraction for linear systems subject to actuator saturation. IEEE Trans. Aut. Cont., 47(1):140-145, 2002.

[2] Y.Y. Cao, Z. Lin, and D.G. Ward. Antiwindup design for linear systems subject to input saturation. Journal of guidance navigation and control, 25(3):455-463, 2002.

[3] E.B. Castelan, I. Queinnec, S. Tarbouriech, and J.M. Gomes da Silva Jr. LMI approach for $\uparrow_{2}$-control of linear systems with saturating actuators. In NOLCOS, pages 287-292, Stuttgart, Germany, 2004.

[4] S. Crawshaw and G. Vinnicombe. Anti-windup synthesis for guaranteed $\mathcal{L}_{2}$ performance. In Proceedings of the Conference on Decision and Control, pages 1063-1068, Sidney, Australia, December 2000.

[5] S. Crawshaw and G. Vinnicombe. Anti-windup for local stability of unstable plants. In American Control Conference, pages 645-650, Anchorage (AK), USA, May 2002.

[6] S. Crawshaw and G. Vinnicombe. Anti-windup synthesis for guaranteed $l_{2}$ performance. In American Control Conference, pages 657661, Anchorage (AK), USA, May 2002.

[7] J.M. Gomes da Silva Jr and S. Tarbouriech. Anti-windup design with guaranteed regions of stability: an LMI-based approach. In Conference on Decision and Control, pages 4451-4456, Maui (HI), USA, December 2003.

[8] C. Edwards and I. Postlethwaite. An anti-windup scheme with closed-loop stability considerations. Automatica, 35(4):761-765, 1999.

[9] G. Grimm, J. Hatfi eld, I. Postlethwaite, A.R. Teel, M.C. Turner, and L. Zaccarian. Antiwindup for stable linear systems with input saturation: an LMI-based synthesis. IEEE Trans. Aut. Cont. (A), 48(9):1509-1525, September 2003.

[10] G. Grimm, A.R. Teel, and L. Zaccarian. Linear LMI-based external anti-windup augmentation for stable linear systems. Automatica (B), 40(11):1987-1996, 2004.

[11] G. Grimm, A.R. Teel, and L. Zaccarian. Robust linear anti-windup synthesis for recovery of unconstrained performance. Int. J. Robust and Nonlinear Control (A), 14(13-15):1133-1168, 2004.

[12] T. Hu, Z. Lin, and B.M. Chen. Analysis and design for linear discretetime systems subject to actuator saturation. Systems and Control Letters, 45(2):97-112, 2002.

[13] T. Hu, Z. Lin, and B.M. Chen. An analysis and design method for linear systems subject to actuator saturation and disturbance. Automatica, 38(2):351-359, 2002.

[14] T. Hu, A.R. Teel, and L. Zaccarian. Nonlinear $\mathcal{L}_{2}$ gain and regional analysis for linear systems with anti-windup compensation. In American Control Conference, Portland (OR), USA, June 2005.

[15] S. Miyamoto and G. Vinnicombe. Robust control of plants with saturation nonlinearity based on coprime factor representation. In 36th CDC, pages 2838-2840, Kobe, Japan, December 1996.

[16] E.F. Mulder and M.V. Kothare. Synthesis of stabilizing antiwindup controllers using piecewise quadratic Lyapunov functions. In Proceedings of the American Control Conference, pages 3239-3243, Chicago (IL), June 2000.

[17] E.F. Mulder, M.V. Kothare, and M. Morari. Multivariable anti-windup controller synthesis using linear matrix inequalities. Automatica, 37(9):1407-1416, September 2001.

[18] A.R. Teel and N. Kapoor. The $\mathcal{L}_{2}$ anti-windup problem: Its defi nition and solution. In Proc. 4th ECC, Brussels, Belgium, July 1997.

[19] F. Wu and B. Lu. Anti-windup control design for exponentially unstable LTI systems with actuator saturation. Systems and Control Letters, 52(3-4):304-322, 2004.

[20] L. Zaccarian and A.R. Teel. A common framework for anti-windup, bumpless transfer and reliable designs. Automatica (B), 38(10):17351744, 2002. 